\title{
MoRfología Y DISTRIBUCIÓN DE DOLOTORTULA MNIIFOLIA Y TRACHYPHYLLUM DUSENII (BRYOPHYTA) EN PANAMÁ
}

\author{
JOSÉ GUDIÑO LEDEZMA y NORIS SALAZAR ALLEN¹
}

\begin{abstract}
Summary: Morphology and distribution of Dolotortula mniifolia and Trachyphyllum dusenii (Bryophyta) in Panama. The genus Dolotortula, and the species $D$. mniifolia, are reported for the first time for Panama. Branched stems and multistratose yellow leaf border with 5-6 rows of stereids (in cross section) are described for the first time for the Central American populations of $D$. miniifolia. The morphology and geographical distribution of Trachyphyllum and the species T. dusenii, recently reported for Panama, are revised and updated. Perichaetial leaves with weakly serrulate margins are described for the first time for T. dusenii. The report of this species in Panama represents the northernmost distribution known for the species in tropical America and includes the only populations known for the species in the pacific coast. An updated geographical distribution for both species is presented.
\end{abstract}

Key words: Central America, Dolotortula, morphology, mosses, Neotropic, Panama, Trachyphyllum.

\begin{abstract}
Resumen: El género Dolotortula y la especie $D$. mniifolia, se registran por primera vez para Panamá. Se describen por primera vez para las poblaciones de Centroamérica de $D$. mniifolia tallos ramificados y borde de la hoja de color amarillo, éste en corte transversal, con 5-6 hileras de células de paredes engrosadas. Se detalla y amplía la morfología y distribución geográfica de Trachyphyllum y la especie T. dusenii, recientemente reportados para Panamá. Se reportan por primera vez hojas periqueciales débilmente serruladas para esta especie. La distribución de esta especie en Panamá es la más septentrional conocida para la América tropical, e incluye las únicas poblaciones del género conocidas para la vertiente del Pacífico. Se incluyen mapas con la distribución geográfica actualizada para las dos especies.
\end{abstract}

Palabras clave: Centroamérica, Dolotortula, musgos, morfología, Neotrópico, Panamá, Trachyphyllum.

\section{INTRODUCCIÓN}

La flora de briofitas de Panamá es una de las más diversas de Centroamérica con aproximadamente 1200 especies (Dauphin et al., 2015). A mediados del siglo XX, Crum \& Steere (1950) registraban 31 especies nuevas de musgos que sumaban un total de 134 especies de briofitas registradas para el país. Casi dos décadas después, Crosby (1969) publica un listado de 213 musgos. En este indicaba que la diversidad debería ser mayor. Delgadillo (1994),

1 Smithsonian Tropical Research Institute, Box 084303092. Balboa, Ancon; Republic of Panama. gudinoj@ si.edu, salazarn@si.edu. incrementa la cifra a 309 especies y para 1997 , Salazar Allen \& Chung estimaron que se conocían más de 600 especies. En la actualidad, la diversidad de musgos (ca. 751 especies), ocupa un segundo lugar después de la de México (973 especies) (Salazar Allen \& Gudiño, 2014) pero se continúan encontrando nuevas adiciones a esta flora.

En este trabajo se reporta y describe por primera vez para la República de Panamá el género Dolotortula R.H. Zander, con la especie D. mniifolia (Sull.) Zander. También se detalla la morfología y se amplía la distribución geográfica de las poblaciones de Trachyphyllum A. Gepp y la especie T. dusenii (Müll. Hal. ex Broth.) Broth., recientemente reportados para Panamá (CarvalhoSilva et al., 2014). 


\section{Materiales y Métodos}

Los especímenes estudiados fueron colectados en las provincias de Coclé, Darién y Panamá, entre 100 a $800 \mathrm{msnm}$. Se utilizaron varias claves taxonómicas en la determinación de ambos géneros y especies (Buck, 1979; Buck \& Griffin, 1984; Zander, 1989, 1993; Mishler, 1994; Churchill \& Linares, 1995; Churchill \& Salazar Allen, 2001; Allen, 2002). Muestras de Trachyphyllum se enviaron al Dr. William R. Buck para su verificación.

Se realizó un estudio morfológico detallado de las especies encontradas. Se tomaron fotografías de los caracteres morfológicos y anatómicos de las plantas con una cámara digital Olympus DP25 incorporada a un microscopio Olympus BX50.

Microscopia electrónica de barrido (SEM) fue utilizada para la observación más detallada de las papilas en la hoja de T. dusenii. Para ello, las muestras secas de la planta fueron rehidratadas parcialmente en Etanol: $\mathrm{H}_{2} \mathrm{O}$ destilada $50: 50$ por 30 minutos, sumergidas en etanol al $70 \%$ por otros 30 minutos y posteriormente se maceraron en nitrógeno líquido (rompimiento por congelación). Trozos y secciones de las hojas fueron colectados en una canasta miliporo de polipropileno y se pasaron por una batería de deshidratación con etanol al 70-80-90$100 \%$ (10 minutos en cada solución). Las muestras fueron secadas al punto crítico en un secador Denton Vacuum CPD 1 y recubiertas con una aleación de oro:paladio en un cobertor iónico Sputtering System Hummer VIA (Anatech Ltd.). Las muestras recubiertas fueron observadas y fotografiadas en un microscopio electrónico de barrido Zeiss Evo $40 \mathrm{vp}$, utilizando un detector de electrones retro-dispersos y $25 \mathrm{kv}$ (voltaje de aceleración).

Se realizó una revisión bibliográfica de la distribución geográfica de D. mniifolia y $T$. dusenii, para generar mapas actualizados de sus respectivas distribuciones en el Neotrópico.

\section{Resultados}

Dolotortula mniifolia (Sull.) Zander. Phytologia 65: 426. 1989. Fig. 1

Plantas de color verde oscuro a parduzco, creciendo en parches laxos. Tallos erectos, 3-5 $\mathrm{mm}$ de alto; cordón central presente pero poco desarrollado. Hojas más o menos dispuestas en roseta, erectas o extendidas; oblongas a oblongoliguladas, con ápices redondos o ligeramente apiculados; planas cuando húmedas, retorcidas cuando secas; 0.9-1.2 $\mathrm{mm}$ de ancho, 2.8-3.4 mm de largo; lámina uni-estratosa; margen entero, con borde multi-estratoso de estereidas amarillo a rojizo que bordea casi por completo la hoja; costa subpercurrente a percurrente; células apicales lisas, principalmente hexagonales, 13.4-21.3 × 17.9-38.5 $\mu \mathrm{m}$, paredes firmes, no porosas; células basales lisas, generalmente rectangulares y alargadas, a veces cuadradas, $20.6-32.4 \times 35.5-109.7 \mu \mathrm{m}$, paredes firmes, no porosas; células alares no diferenciadas. Plantas dioicas. Periquecio terminal. Perigonio no observado. Esporofito no observado.

Material examinado. PANAMÁ. Prov. de Coclé: Dtto. Antón, El Valle de Antón, La Pintada, subiendo por el Cerro La India Dormida, 02XI-2007, Gudiño 396, (PMA). Prov. Darién: Dtto. Chepigana, Reserva Natural Privada Cerro Chucantí, 22-XII-2009, Gudiño 924, 927C (PMA).

Hábitat. En el Valle de Antón, las plantas de $D$. mniifolia crecen sobre una capa delgada de suelo en roca húmeda, muy cerca de una pequeña caída de agua. En el Cerro Chucantí, la especie crece junto a plantas de Hyophila involuta (Hook.) A. Jaeger (Pottiaceae), en un sitio perturbado, sobre suelo desnudo, alrededor de la casa de hospedaje de la reserva privada que se ha establecido en el cerro. Desconocemos si esta población fue introducida al lugar, o si es originaria de esta zona.

Observaciones. Dolotortula (Pottiaceae) es un género monotípico, con $D$. mniifolia como única representante. Originalmente la especie fue incluida en el género Tortula Hedw., como T. mniifolia (Bartram, 1949; Crum \& Steere, 1958; Welch \& Crum, 1959; Pursell, 1973), pero fue separada de éste y colocada en un género independiente por Zander (1989).

Dolotortula forma parches laxos, de color verde oscuro (Fig. 1A). A primera vista la planta se asemeja a una Mniaceae o Bryaceae, debido a sus hojas limbadas (Fig. 1C, G-H). Pero se diferencia fácilmente de los miembros neotropicales de estas familias, por sus hojas de bordes lisos (sin dientes) y borde multiestratoso (Fig. 1I) (Churchill \& 



Fig. 1. Dolotortula mniifolia (Sull.) Zander. A. Hábito. B. Corte transversal del tallo. C. Hoja. D. Células apicales de la hoja. E. Células basales de la hoja. F. Corte transversal de la nervadura. G-H. Borde multiestratoso de la hoja. I. Corte transversal del borde de la hoja, mostrando 5-6 estratos. A, B, D-I = Gudiño 396; C = Gudiño 927C. Escala: $A=9 \mathrm{~mm} ; B=0.1 \mathrm{~mm} ; \mathrm{C}=0.6 \mathrm{~mm} ; \mathrm{D} \& \mathrm{~F}=30 \mu \mathrm{m} ; \mathrm{E}=50 \mu \mathrm{m} ; \mathrm{G}-\mathrm{H}=40$ $\mu \mathrm{m} ; \mathrm{l}=9 \mu \mathrm{m}$. 
Salazar Allen, 2001; Allen, 2002). Estos caracteres, junto a células de la lámina lisas (Fig. 1D-E), también ayudan a diferenciar a Dolotortula de los demás géneros de la familia Pottiaceae (Zander 1989, 1993; Allen, 2002).

En Centroamérica, Allen (2002) atribuye a $D$. mniifolia plantas con tallos no ramificados; sin embargo, en la población de El Valle de Antón, algunos individuos presentaron tallos con una ramificación. Esta morfología coincide con la descripción de Zander (1989) que describe el tallo como "seldom branching" o sea, ocasionalmente ramificado.

Allen (2002) indica que el borde multriestratoso de la hoja en los especímenes de Centro América puede tener hasta 4 estratos. Sin embargo, en las poblaciones panameñas el borde de la hoja está compuesto por 28-50 hileras de células dispuestas en (4-) 5-6 estratos (Fig. 1I). En este aspecto se asemejan a las poblaciones mexicanas descritas por Mishler (1994).

Allen (2002) señala que el borde multiestratoso es de color rojo; en las colectas panameñas esta característica es variable. En la población de El Valle de Antón, las plantas presentan bordes generalmente amarillentos (Fig. 1G). Bordes de color rojizo a rojo intenso se observaron exclusivamente en las hojas viejas (Fig. 1H), típicamente con la lámina foliar deteriorada; en los casos más extremos, el borde multiestratoso permanece unido al tallo, mientras que el resto de la hoja se ha descompuesto. Sin embargo, los especímenes de Cerro Chucantí concuerdan con la descripción de Allen (2002), con bordes de color anaranjado a rojo intenso, tanto en hojas jóvenes como viejas.

La coloración del borde multiestratoso en los especímenes de Cerro Chucantí podría indicar que la misma estaba formada por especímenes más viejos que los encontrados en El Valle de Antón. En Dolotortula, esta pigmentación se debe esencialmente a pigmentos presentes en las paredes celulares. En muchos tejidos de las briofitas, la pigmentación en la pared celular tiende a aumentar a medida que la planta envejece (Mårtensson \& Nilsson, 1974). Sin embargo, factores microambientales también podrían explicar el porqué de esta diferencia en coloración. Los especímenes colectados en el Cerro Chucantí se encontraron en un sitio más seco y expuesto que los colectados en El Valle de Antón. En hepáticas foliosas se ha demostrado que pigmentos asociados a las paredes celulares pueden ayudar a mitigar daños celulares producto de altas intensidades lumínicas y aumentar la tolerancia a la desecación (Hooijmaijers \& Gould, 2007; Hooijmaijers, 2008).

Distribución. Dolotortula mniifolia es una especie endémica del Neotrópico (Churchill \& Linares, 1995; Delgadillo et al., 1995; Churchill \& Salazar Allen, 2001). Aunque es considerada como una especie poco común, o "rara", tiene una amplia distribución en las Américas (Fig. 2). Ha sido reportada para México (Mishler, 1994), Guatemala (Bartram, 1949; Allen, 2002; Salazar et al., 2006), Honduras, Costa Rica (Allen, 2002), Cuba (Mishler, 1994; Potrony et al., 2007), Jamaica (Welch \& Crum, 1959; Schäfer-Verwimp \& Van Melick, 2016), Haití (Crum \& Steere, 1958; Mishler, 1994), República Dominicana (Sastre-D. J. et al., 2010), Puerto Rico (Sastre-D.J. \& Buck, 1993), Trinidad \& Tobago (Mishler, 1994; Delgadillo et al., 1995), Colombia (Churchill \& Linares, 1995; Ramírez \& Churchill, 2002; Santos-C. \& Aguirre, 2010), Venezuela (Pursell \& Curry, 1969; Pursell, 1973; O’Shea, 2010), Ecuador (Churchill et al., 1992; Churchill, 1998), Perú (Churchill, 1998; Churchill et al., 2000), Brasil (Costa et al., 2011; Costa, 2016), Bolivia (Churchill et al., 2000; Fuentes \& Churchill, 2005) y Argentina (Suárez et al., 2005).

En esta publicación, el género Dolotortula y la especie D. mniifolia se reportan por primera vez para Panamá, para las provincias de Coclé (El Valle de Antón) y Darién (Cerro Chucantí).

Trachyphyllum dusenii (Müll. Hal. ex Broth.) Broth. Die Natürlichen Pflanzenfamilien I(3): 890. 1907. Fig. 3-4

Plantas rastreras, de color verde amarillento a dorado opaco, creciendo en parches densos; tallos rastreros. Ramas densamente terete-foliadas (a veces ligeramente complanadas). Hojas extendidas o escuarrosas, ligeramente aplanadas a cóncavas, anchamente lanceoladas, 0.2-0.4 $\mathrm{mm}$ de ancho y 0.5-1.0 mm de largo; márgenes planos, lisos o ligeramente serrulados hacia el ápice; costa corta y doble; células apicales de la lámina alargadas o fusiformes, prorulosas, paredes firmes, no porosas, 




Fig. 2. Distribución de Dolotortula mniifolia (Sull.) Zander.

4-5(-7) × 22-45 $\mu \mathrm{m}$; células basales fusiformes, alargadas o rectangulares, comúnmente prorulosas, paredes firmes, no porosas, 4-5 × 14-40 $\mu \mathrm{m}$; células alares numerosas 15->30, cuadradas o rectangulares (a veces triangulares), extendiéndose hacia los márgenes de la hoja, con paredes celulares firmes, no porosas, 4-9(-13) × 9-11 $\mu \mathrm{m}$. Plantas dioicas. Perigonio no observado. Periquecio lateral; hojas largas triangulares lanceoladas, gradual y largamente acuminadas, y hojas cortas generalmente con bases ovadas, abruptamente acuminadas; $1.2-2.0 \mathrm{~mm}$ de largo, 0.19-0.34 mm de ancho; márgenes lisos o débilmente serrulados hacia el ápice; costa ausente, simple o doble, extendiéndose hasta $1 / 2$ de la hoja; células alargadas, rectangulares hacia el 1/4-1/3 basal, gradualmente tornándose fusiformes hacia el ápice. Esporofitos maduros no observados.
Material examinado. PANAMÁ. Prov. Coclé: Dtto. Antón, El Valle de Antón, Monumento Natural Cerro Gaital, Río Guayabo, 17-I-2009, Gudiño 599 (PMA). Prov. Darién: Dtto. Chepigana, Reserva Natural Privada Cerro Chucantí, 22-XII-2009, Gudiño 876 A, 905B, 929, 934 (PMA); 03-III-2011, Vergara 304 (PMA). Prov. Panamá: Dtto. Panamá, Parque Nacional Chagres, Campo Chagres, sendero Las Grietas, 16-XI-2012, Gudiño 1542 (PMA).

Hábitat. En el Cerro Chucantí (Darién), la especie es relativamente común, encontrándose en troncos en descomposición y en rocas de río. Mientras que, en El Valle de Antón (Coclé) sólo se encontró un espécimen sobre tronco en descomposición, en medio del cauce del Río Guayabo; en Campo Chagres (Panamá) se colectó un único espécimen creciendo sobre corteza viva, en la base de un árbol. El género Trachyphyllum 
Bol. Soc. Argent. Bot. 52 (2) 2017
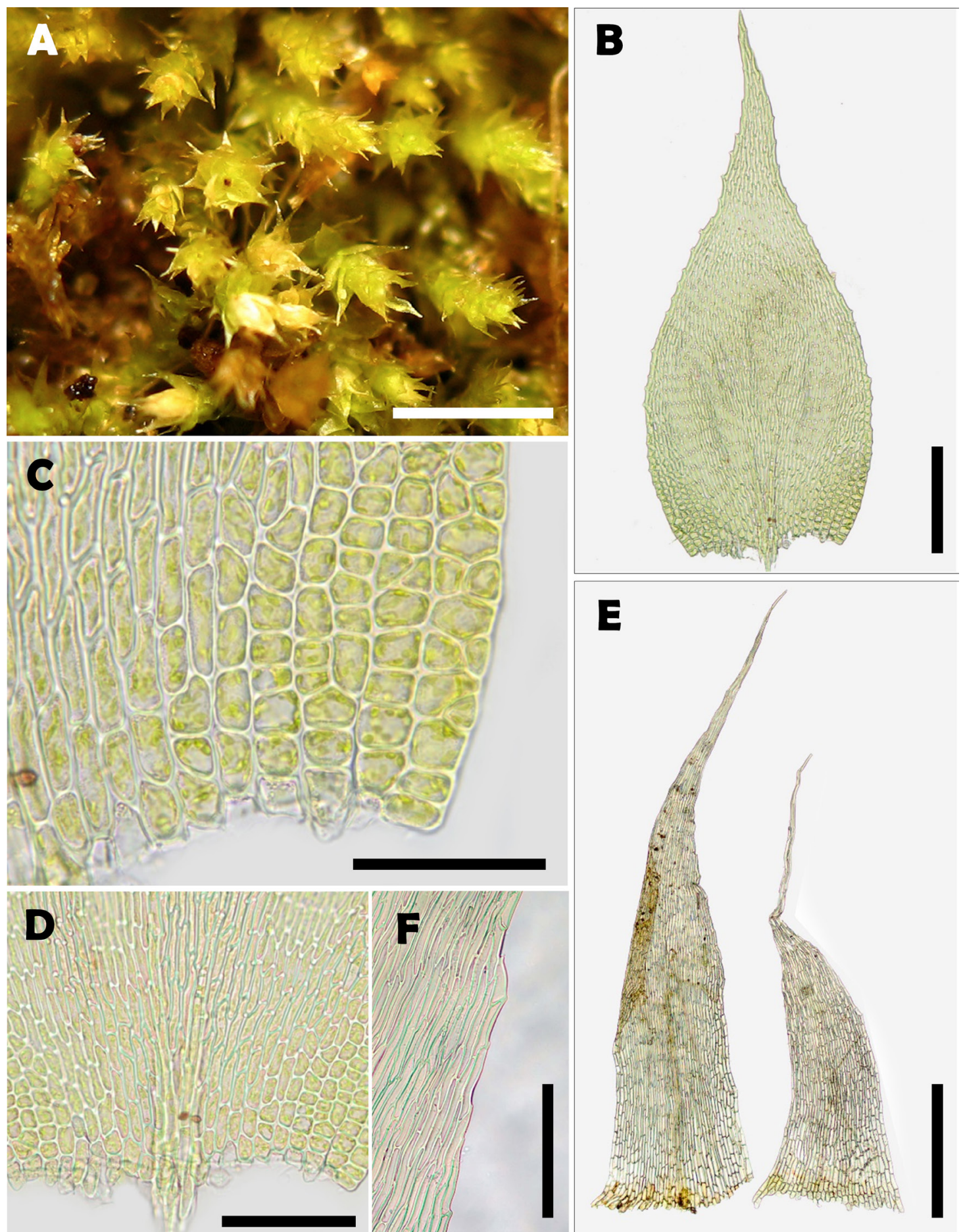

Fig. 3. Trachyphyllum dusenii (Müll. Hal. ex Broth.) Broth. A. Hábito. B. Hoja. C. Células alares de la hoja. D. Costa de la hoja. E. Hojas periqueciales. F. Margen distal de la hoja periquecial. A-E = Gudiño 599; F-G $=$ Vergara 304. Escalas: $A=1.5 \mathrm{~mm} ; B=0.18 \mathrm{~mm} ; C=40 \mu \mathrm{m} ; \mathrm{D}=81 \mu \mathrm{m} ; E=0.38 \mathrm{~mm} ; \mathrm{F}=95 \mu \mathrm{m}$. 



Fig. 4. Hoja papilosa de Trachyphyllum dusenii (Müll. Hal. ex Broth.) Broth. A. Papilas en microscopia de luz. B. Papilas en microscopia electrónica. C. Micrografía electrónica de la sección transversal de la hoja y las papilas. $A=$ Gudiño 599; B-C = Gudiño 876A. Escala: $A=29$ $\mu \mathrm{m} ; \mathrm{B}=19 \mu \mathrm{m} ; \mathrm{C}=8 \mu \mathrm{m}$. se desarrolla generalmente en áreas sombreadas (Churchill \& Salazar Allen, 2001), sin embargo, en Cerro Chucantí, los especímenes fueron colectados comúnmente en claros de bosque.

Observaciones. Carvalho-Silva et al. (2014) reportaron la especie por primera vez para Panamá en base al espécimen colectado por nosotros en El Valle de Antón (Gudiño 599). Inicialmente, éste y otros especímenes de $T$. dusenii se ubicaron en el género Chryso-hypnum Hampe, por sus hojas con costa doble y corta (Fig. 3B), y células de la lámina fuertemente prorulosas (Fig.4). Sin embargo, el color verde amarillento, ramas terete-foliadas (Fig. 3A) y las hojas \pm cóncavas, anchamente lanceoladas, con un acumen largo y una región alar conspicua (Fig. 3C), no coincidían con los caracteres de Chryso-hypnum.

Observaciones de un espécimen de T. gastrodes (Welw. \& Duby) A. Gepp, del Herbario de la Universidad de Panamá (PMA), ayudaron a determinar que los especímenes colectados correspondían al género Trachyphyllum. Los especímenes Gudiño 599 (El Valle de Antón) y 876A (Cerro Chucantí) fueron enviados al especialista del género, Dr. William R. Buck, quien confirmó el género y determinó la especie, T. dusenii.

En el Cerro Chucantí, T. dusenii con frecuencia crece entremezclada con especies de la familia Hypnaceae, Ectropothecium leptochaeton (Schwägr.) W.R. Buck. y Chryso-hypnum diminutivum (Hampe) W.R. Buck. Ectropothecium leptochaeton se diferencia fácilmente de $T$. dusenni por sus hojas falcado-secundas, células lisas y región alar poco desarrollada. A pesar que algunos caracteres de la anatomía foliar de Trachyphyllum son similares a los de Chryso-hypnum, el hábito de T. dusenii contrasta fuertemente con el de $C$. diminutivun, al menos en las poblaciones panameñas.

Las plantas de T. dusenii son más robustas, con ramas teretes a ligeramente complanadas, y densamente foliadas; las de $C$. diminutivum son más pequeñas y flexuosas, con ramas laxamente foliadas y hojas más o menos complanadas. Trachyphyllum dusenii además presenta hojas cóncavas, con una región alar bien diferenciada (más o menos triangular), formada por células cuadradas que se extiende de (6-)911(-15) células en el margen de la hoja, y de 4(-6) células de ancho en la base de la hoja. Chryso-hypnum diminutivum presenta hojas aplanadas y carece de una región alar conspicua, frecuentemente con 3-5 
células cuadradas en los flancos de la hoja.

No se encontraron esporofitos maduros, sin embargo en el Cerro Chucantí se observaron especímenes de $T$. dusenii (Gudiño 876A, 905B y Vergara 304) con esporofitos inmaduros. De acuerdo a los estados de desarrollo de la cápsula propuestos por Greene (1960), los esporofitos observados se encuentran en estado tardío "caliptra en periquecio" (LCP, por sus siglas en inglés). Buck (1979) y Buck \& Griffin (1984) describen las hojas periqueciales de T. dusenii como "muy serradas, a veces con dientes multicelulares y ampliamente extendidos a reflexos". Sin embargo, en los especímenes panameños, los esporofitos LCP estaban rodeados por hojas periqueciales débilmente serruladas hacia la porción distal de la hoja, con ápices de las células marginales apenas proyectándose del margen (Fig. 3E-F).
Distribución. El género Trachyphyllum se distribuye principalmente en el viejo mundo (Buck, 1979; Buck \& Griffin, 1984): África, India, Sudeste de Asia, Filipinas y el norte de Australia. Trachyphyllum dusenii tiene una distribución estrictamente tropical. En África, su distribución parece ser interrumpida por el desierto del Sahara, siendo Senegal la localidad más septentrional conocida de la especie (Buck, 1979).

Trachyphyllum y la especie $T$. dusenii fueron reportados por primera vez para el Neotrópico por Buck \& Griffin (1984). En esta región, la especie se distribuye principalmente en Sudamérica (Fig. 5), encontrándose en Guyana (Carvalho-Silva et al., 2014), Brasil (Buck \& Griffin, 1984; Churchill, 1998; Costa et al., 2011), Bolivia (Churchill et al., 2000; Churchill \& Fuentes C., 2005) y Argentina (Ochyra, 1999; Matteri, 2003).

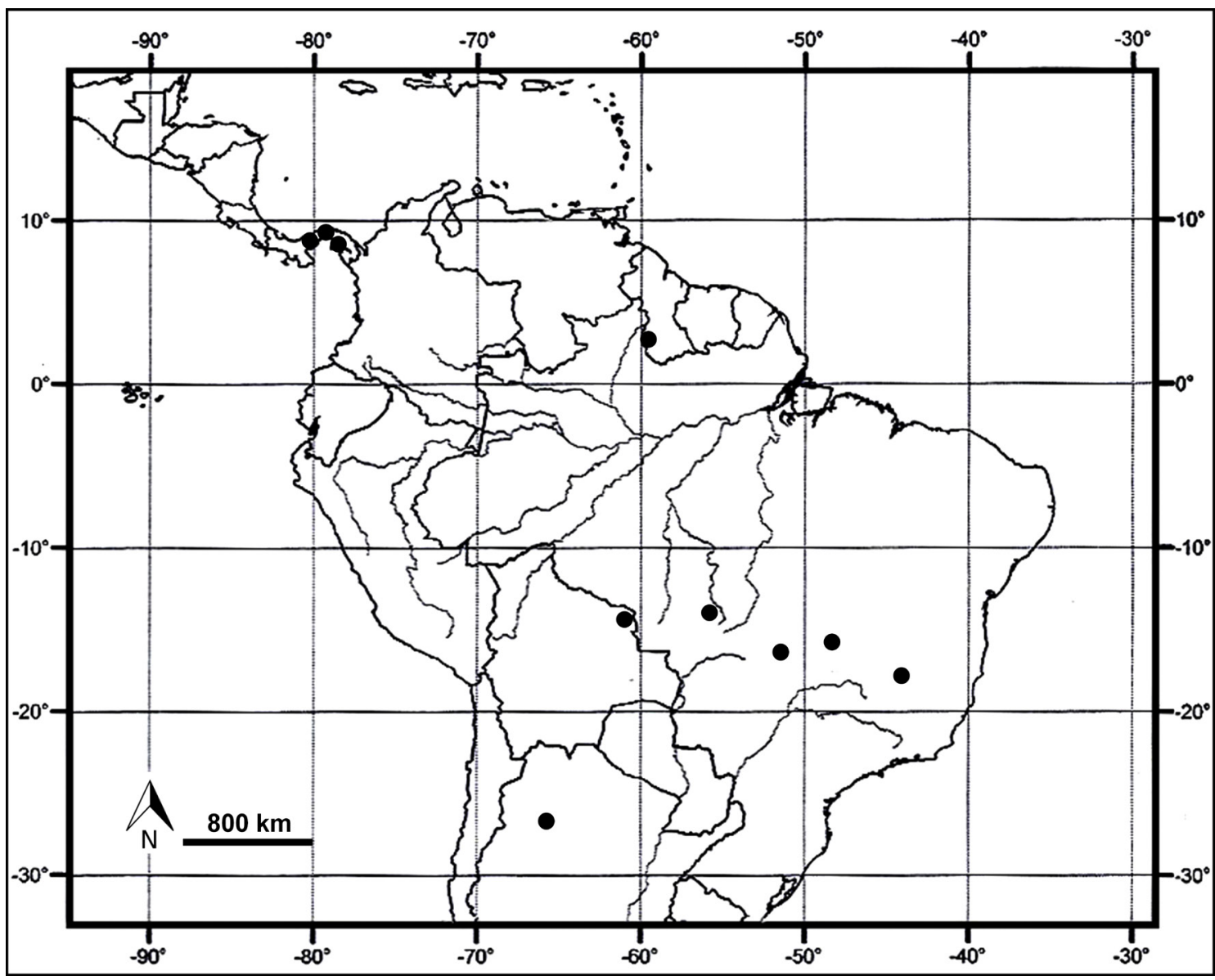

Fig. 5. Distribución de Trachyphyllum dusenii (Müll. Hal. ex Broth.) Broth. en el Neotrópico. 


\section{J. Gudiño Ledezma y N. Salazar Allen - Dolotortula mniifolia y Trachyphyllum dusenii}

El registro de Trachyphyllum, así como de $T$. dusenii, para Panamá (Carvalho-Silva et al., 2014) es el primer y único registro para Centroamérica, encontrándose en las provincias de Darién (Cerro Chucantí), Panamá (Campo Chagres) y Coclé (El Valle de Antón). Panamá es la localidad más septentrional hasta ahora conocida para la especie en la América Tropical. Esto sugiere que la especie puede tener una distribución mucho más amplia en Sudamérica que la conocida actualmente.

Las poblaciones panameñas de T. dusenii (principalmente las de Cerro Chucantí) actualmente son las únicas conocidas para la vertiente del Pacífico. Aparte de Panamá, Trachyphyllum dusenii sólo es conocida en localidades de la vertiente atlántica y la región central de Sudamérica (Fig. 5), así como en África (Buck, 1979; Buck \& Griffin, 1984; Carvalho-Silva et al., 2014).

Trachyphyllum dusenii es uno de los numerosos ejemplos de especies con distribución disjunta entre América y África (Buck \& Griffin, 1984; Delgadillo, 1993), al igual que C. diminutivum (Delgadillo \& Buck, 1988). Es muy probable que por el origen reciente (Mioceno) del Istmo de Panamá, estas especies, así como otras más (e.g., Leucophanes molleri Müll. Hal.) tuvieron su origen en las poblaciones de Sudamérica (Salazar Allen et al., 1991).

\section{Agradecimientos}

Agradecemos especialmente al Dr. William R. Buck por la determinación de la especie $T$. dusenii; al Lic. Guido Berguido (fundador de la Reserva Natural Privada Cerro Chucantí) y a la Dra. Alicia Ibáñez por facilitar la visita y estadía de José Gudiño L. en la Reserva, así como a los guías de la Reserva; al Lic. Roberto Vergara por sus colecciones de la Reserva; a la Lic. Adhara y Atria de la Barrera por su guía durante la colecta de Dolotortula en el Valle de Antón; al Lic. Jorge Ceballos, técnico del Laboratorio de Microscopia Electrónica y Confocal del Instituto Smithsonian de Investigaciones Tropicales (STRI), por su colaboración en la toma de micrografías de T. dussenii y, al STRI por financiamiento para las investigaciones de N. Salazar Allen sobre las briofitas de Panamá y para su técnico de investigación José Gudiño L.

\section{Bibliografía}

ALLEN, B. H. 2002. Moss Flora of Central America, Part 2 (Encalyptaceae-Orthotrichaceae). Monogr. Syst. Bot. Missouri Bot. Gard. 49. Missouri Botanical Garden Press. St. Louis, USA.

BARTRAM, E. B. 1949. Mosses of Guatemala. Fieldiana, Bot. 25: 1-442.

BUCK, W. R. 1979. A revision of the moss genus Trachyphyllum Gepp (Thuidiaceae). Brittonia 31(3): 379-394.

BUCK, W. R. \& D. GRIFFIN III. 1984. Trachyphyllum, a moss genus new to South America with notes on African-South American bryogeography. J. Nat. Hist. 18: 63-69.

CARVALHO-SILVA, M., T. DA SILVA DANTAS \& P. E. A. S. CÂMARA. 2014. Systematic position of the moss genus Trachyphyllum A. Gepp (Pylaisiadelphaceae) plus additions to its distribution in the Neotropics. J. Bryol. 36: 217-222.

CHURCHILL, S. P., I. SASTRE-DE JESÚS \& H. BALSLEV. 1992. The mosses of Añangu, Napo Province, Ecuador. Lindbergia 17: 50-54.

CHURCHILL, S. P. \& E. L. C. LINARES. 1995. Prodromus bryologiae Novo-Granatensis. Introducción a la flora de musgos de Colombia. Inst. Cs. Nat.-Mus. Hist. Nat. Bibliot. «Jose J. Triana»12, parte 2. Santa Fe de Bogotá.

CHURCHILL, S. P. 1998. Catalog of Amazonian mosses. J. Hattori Bot. Lab. 85: 191-238.

CHURCHILL, S. P., D. GRIFFIN III \& J. MUÑOZ. 2000. A checklist of the mosses of the tropical Andean countries. Ruizia 17: 1-303.

CHURCHILL, S. P. \& N. SALAZAR ALLEN. 2001 Mosses. En: Gradstein, S.R., S. Churchill \& N. Salazar Allen. A guide to the bryophytes of Tropical America. Memoir New York Bot Gard 86, New York. USA.

CHURCHILL, S. P. \& A. FUENTES C. 2005. Additions, Combinations, and Synonyms for the Bolivian Moss Flora. Trop. Bryol. 26: 119-131.

COSTA, D. P., K. C. PÔRTO, A. P. LUIZI-PONZO, A. L. ILKIU-BORGES, C. J. P. BASTOS, P. E. A. S. CÂMARA, D. F. PERALTA, S. B. V. BÔAS-BASTOS, C. A. A. IMBASSAHY, D. K. HENRIQUES, H. C. S. GOMES, L. M. ROCHA, N. D. SANTOS, T. S. SIVIERO, T. F. VAZIMBASSAHY \& S. P. CHURCHILL. 2011. Synopsis of the Brazilian moss flora: checklist, distribution and conservation. Nova Hedwigia 93(3-4): 277-334.

COSTA, D. P. DA. 2016. A Synopsis of the family Pottiaceae in Brazil. Phytotaxa 251(1): 001-069.

CROSBY, M. R. 1969. The mosses reported from Panamá. Bryologist 72(4): 513-521. 
CRUM, H. A. \& W. C. STEERE. 1950. Additions to the moss flora of Panama. Bryologist 53(2): 139-152.

CRUM, H. A. \& W. C. STEERE. 1958. A contribution to the bryology of Haiti. Am. Midl. Nat. 60(1): $1-51$.

DAUPHIN, G., N. SALAZAR ALLEN, J.A. GUDIÑO, A. SIERRA \& D. REYES. 2015. Nuevas adiciones de especies de hepáticas (Marchantiophyta) para la flora de Panamá. II. Brenesia 83-84: 16-21.

DELGADILLO M., C. 1993. The Neotropical-African moss disjunction. Bryologist 96(4): 604-615.

DELGADILLO M., C. 1994. Endemism in the Neotropical moss flora. Biotropica 26(1): 12-16.

DELGADILLO M., C. \& W. R. BUCK. 1988. Levierella (Fabroniaceae), a moss genus new to the Americas. Bryologist 91(1): 53-55.

DELGADILlO M., C., B. BELLO \& A. CÁRDENAS. 1995. Latmoss. A catalogue of Neotropical mosses. Monogr. Syst. Bot. Missouri Bot. Gard. 56: 1-191.

FUENTES, A. \& S. P. CHURCHILL. 2005. Catálogo preliminar de las briófitas de la región de Madidi, Bolivia. Ecol. Boliv. 40(3): 170-198.

GREENE, S. W. 1960. The maduration cycle or the stages of development of gametangia and capsules in mosses. Trans. Brit. Bryol. Soc. 3: 736-745.

HOOIJMAIJERS, C. A. M. 2008. Desiccation tolerance in red and green gametophytes of Jamesoniella colorata in relation to photoprotection. Planta 227(6): 1301-1310.

HOOIJMAIJERS, C. A. M. \& K. S. GOULD. 2007. Photoprotective pigments in red and green gametophytes of two New Zealand liverworts. N.Z. J. Bot. 45: 451-461.

MÅRTENSSON, O. \& E. NILSSON. 1974. On the morphological colour of bryophytes. Lindbergia 2(3): 145-159.

MATTERI, C. M. 2003. Los musgos (Bryophyta) de Argentina. Trop. Bryol. 24: 33-100.

MISHLER, B. D. 1994. Tortula. En: Sharp, A., H. Crum, \& P. Eckel (eds.), The moss flora of Mexico. Pt. 1. Memoir New York Bot. Gard. 69, New York.

OCHYRA, R. 1999. The identities of three neotropical species of Hygrohypnum (Musci, Amblystegiaceae). Fragm. Florist. Geobot. 44: 261-268.

O'SHEA, B. J. 2010. Mosses of Venezuela. Archive for Bryology 75: 1-23.

POTRONY H. M. E., A. M. MARÍN \& O. J. REYES. 2007. Patrones de distribución y vías de migración de los musgos de la Sierra Maestra. Polibotánica 24: 167-176.

PURSELL, R. A. \& C. L. CURRY. 1969(1970). A contribution to the bryology of Venezuela. Acta Bot. Venez. 4: 69-85.

PURSELL, R. A. 1973. Un censo de los musgos de Venezuela. Bryologist 76(4): 473-500.
RAMÍREZ P., B. R. \& S. P. CHURCHILL. 2002. Las briofitas del Departamento de Nariño, Colombia: I. Musgos. Trop. Bryol. 21: 23-46.

SALAZAR ALLEN., N., C. ARROCHA \& C. CHUNG. 1991. The mosses of Barro Colorado Island, Panama. Bryologist 94(3): 289-293.

SAlAZAR ALlEN., N., \& C. CHUNG C. 1997. Pág. 221- 243. Primera aproximación al catálogo de briófitos del Parque Nacional de Coiba (Panamá). En: Flora y fauna del Parque Nacional de Coiba (Panamá). S. Castroviejo (Eds.). Agencia Española de Cooperación Internacional (AECI). Madrid.

SALAZAR ALLEN, N., J. E. DE GARCÍA \& C. CHUNG. 2006. Aporte al catálogo de musgos de Guatemala. En: Biodiversidad de Guatemala. Enio B. Cano (Ed.) Volumen I. Universidad del Valle de Guatemala. Guatemala. Centro América.

SALAZAR ALLEN, N. \& J. A. GUDIÑO. 2014. Las Briofitas. Jardín de Miniaturas Vegetales. Instituto Smithsonian de Investigaciones Tropicales. Panamá. 4 págs.

SANTOS-C., G. \& J. AGUIRRE-C. 2010. Los musgos de la región de Las Quinchas (Magdalena Medio, Colombia). Caldasia 32(2): 257-273.

SASTRE-D. J., I. \& W. R. BUCK. 1993. Annotated checklist of the mosses of Puerto Rico. Caribb. J. Sci. 29(3-4): 226-234.

SASTRE-D.J., I., M. PEREZ PEREZ \& A. M. MARIN. 2010. Mosses of the Dominican Republic: species catalogue, elevation distribution and floristic affinities. Harvard Pap. Bot. 15(2): 415-446.

SCHÄFER-VERWIMP, A. \& H. M. H. VAN MELICK. 2016. A Contribution to the Bryophyte Flora of Jamaica. Cryptogamie, Bryologie 37(3):305-348.

SUAREZ, G. M., M. T. COLOTTI \& M. M. SCHIAVONE. 2005. Dolotortula mniifolia (Sull.) Zand. (Pottiaceae, Musci) en Argentina. Lilloa 42(12): 81-84.

WELCH, W. H. \& H. CRUM. 1959. A contribution to the Jamaican moss flora. Bryologist 62(3): 165-179.

ZANDER, R. H. 1989. Seven new genera in Pottiaceae (Musci) and a lectotype for Syntrichia. Phytologia 65: 424-436.

ZANDER, R. H. 1993. Genera of the Pottiaceae: mosses of harsh environments. Bull. Buffalo Soc. Nat. Sci. 32: 1-378.

Recibido el 1 de abril de 2017, aceptado el 5 de junio de 2017. 